Recogniti on and cutting system of sweet pepper for pi cking robot in greenhouse horticul ture

\begin{tabular}{|l|l|}
\hline 著者 & K t amur a Shi nsuke, Oka Koi chi \\
\hline vol une & 4 \\
\hline page range & $1807-1812$ \\
\hline year & 2005 \\
\hline URL & ht t p: //hdl . handl e. net /10173/297 \\
\hline
\end{tabular}




\section{Recognition and Cutting System of Sweet Pepper for Picking Robot in Greenhouse Horticulture}

\author{
Shinsuke Kitamura \\ Department of Intelligent Mechanical Systems Engineering \\ Kochi University of Technology \\ 185 Miyanokuchi, Tosayamada-cho, Kochi, 782-8502 Japan \\ 075031e@gs.kochi-tech.ac.jp
}

\begin{abstract}
This paper describes recognition and cutting system of sweet peppers for picking robots in greenhouse horticulture. This picking robot has an image processing system with a parallel stereovision, a camera positioning system to follow the sweet pepper by visual feedback control, and a cutting device. A prototype robot system has been made and is introduced. Experiments of the prototype prove that performance of the cutting system depends on recognition of fruits of sweet peppers. Consequently, the robot has ability for picking sweet peppers.
\end{abstract}

Index Term s- Picking Robot, Image Processing, Recognition, Visual Feedback Control, Stereovision, Sweet Pepper, Greenhouse.

\section{INTRODUCTION}

In Japan, greenhouse horticulture is flourishing as shown in Fig.1. Greenhouses are covered with vinyl sheets, and keep vegetable from farming enemies such as strong wind, bad weather, harmful insect, and so on. Greenhouse horticulture help agricultural products to grow more efficiently and keeps them high quality because of the controlled circumstance. In recent years, the shortage of farming labor force is getting worse because of impact of the falling birthrate and the aging farmers. For the solution of this problem, some automatic greenhouse horticulture systems have been proposed. They are, for example, automatic temperature control system, automatic watering system, automatic picking system, and so on. Automatic temperature system and automatic watering system have been investigated. Picking robots for eggplant and tomato have been also studied [1][2]. However, their picking robots have not been put to practical use in greenhouse horticulture.

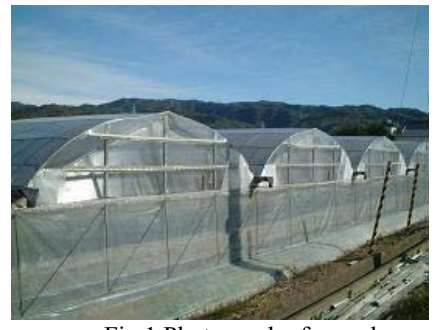

Fig.1 Photograph of greenhouse

\author{
Koichi Oka
}

Department of Intelligent Mechanical Systems Engineering Kochi University of Technology

185 Miyanokuchi, Tosayamada-cho, Kochi, 782-8502 Japan oka.koichi@kochi-tech.ac.jp

The difficulty of picking robots exists in identification of fruits, cutting systems, moving systems, and so on. Recognition of fruits is newly carried out by image processing using camera. Therefore, the picking robot system for the fruit that has special color such as eggplant and tomato has been investigated. Although sweet pepper is major agricultural produce in greenhouse horticulture, its picking robot has not been studied. Because the color of fruits is almost same of leaves, and recognition of the fruit is difficult.

This paper aims development and a feasibility study for the picking robot of sweet peppers using usual image processing technique we constructed the recognition system of sweet peppers and its position, camera positioning system for cutting the stem, and picking robot using these systems. In the results of experiments, if the fruit of the sweet pepper was sharply defined, this robot could cut the stem of the recognized sweet pepper. First, the picking operation for sweet peppers in greenhouse by farmers is introduced. Second, the outline of the picking robot for sweet peppers is explained and prototype of the robot is introduced in detail. Third, the recognition system of sweet peppers control system is explained. Last, the result of experiment is introduced.

\section{Picking robot}

\section{A. Picking operation by farmer}

Sweet pepper trees are planted on chins in greenhouse and sweet peppers are growing on trees as shown in Fig.2. Sweet peppers that are within the standard are picked by farmers. The procedure to pick sweet peppers is follows. First farmers look for sweet peppers that meet the standard of its size/shape with their eyes and hands. They cut stems of the selected sweet peppers, and put into the container as shown in Fig.3.
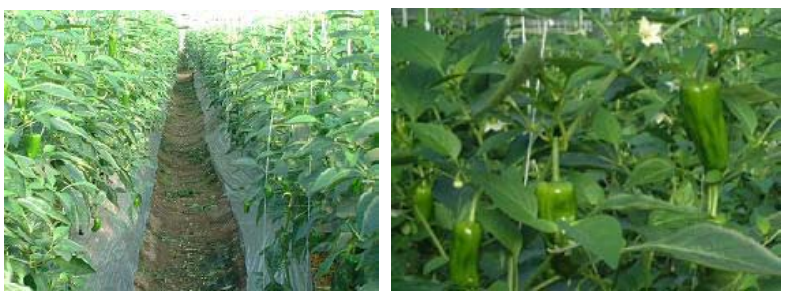

Fig.2 Photograph of sweet pepper growing 

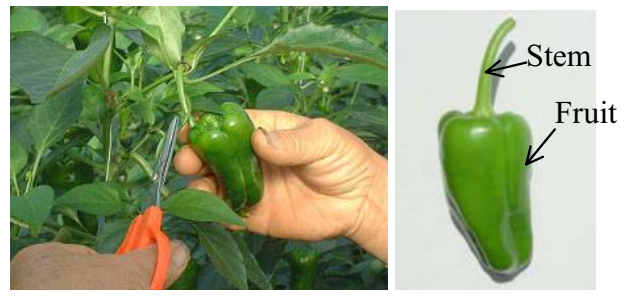

Fig.3 Picking of sweet peppers by farmer

\section{B. Over view}

Our study is the development of picking robot that can recognize sweet peppers, cut the stem, and put the fruit into the container as farmers. Main functions required for this picking robot are shown in Fig.4. They are a recognition technology, a picking technology, and a moving technology. To realize for these functions, the picking robot has an image processing system with a parallel stereovision, a positioning system to follow the recognized sweet pepper by visual feedback control, and a cutting device.

An illustration of a picking robot is shown in Fig.5 and a photo of a prototype robot is shown in Fig.6. This robot is approximately $1000 \mathrm{~mm}$ wide, $550 \mathrm{~mm}$ deep, and $1400 \mathrm{~mm}$ high. When wheels do not move, operating range is $230 \mathrm{~mm}$ in the horizontal direction, $180 \mathrm{~mm}$ in the vertical direction, and $180 \mathrm{~mm}$ in the depth direction. Within the range, it is possible to cut the stems of the recognized sweet peppers. The cutting device is fixed to the frame where two cameras are installed. It moves $80 \mathrm{~mm}$ in the forward direction, and cuts the stem of the recognized sweet pepper by a pruner.

\section{Robot system}

1) Image processing system: The image processing system consists of two color CCD cameras, a capture board, and image processing applications. The image processing system is illustrated in Fig.7. This camera is a high image -quality CCD camera of RF SYSTEM whose specification are 680,000-pixel 1/4inches CCD and 450 TV lines resolution. The image capture board is PicPort of Leutron, and the image processing application is HALCON of MVTec. Two cameras are placed in parallel, detect the position of the sweet pepper can be detected by stereovision method.

2) Camera Positioning System: To cut the stem of the recognized sweet pepper, positioning system uses the feedback loop with cameras for the recognized sweet peppers. In this positioning system, camera can be positioning as it follows the sweet pepper in the three dimensions, horizontal direction, vertical direction, and depth direction. The positioning system has slide rale, timing belt, and DC motor in each dimension. All motors controlled by DSP controller. Image processing system provides the information of the position of the sweet pepper to the controller. Based on the information the positioning system controls the camera position. The positioning system of the camera is shown in Fig.8.

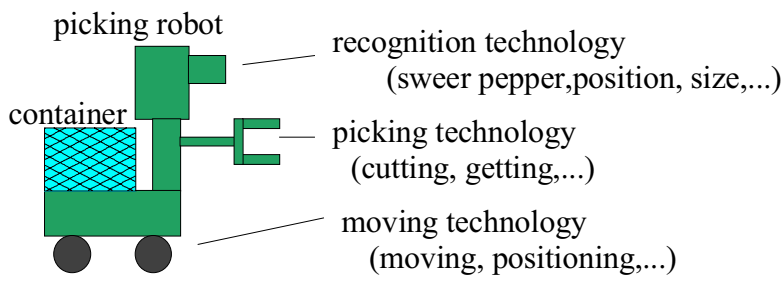

Fig.4 Concept for picking robot

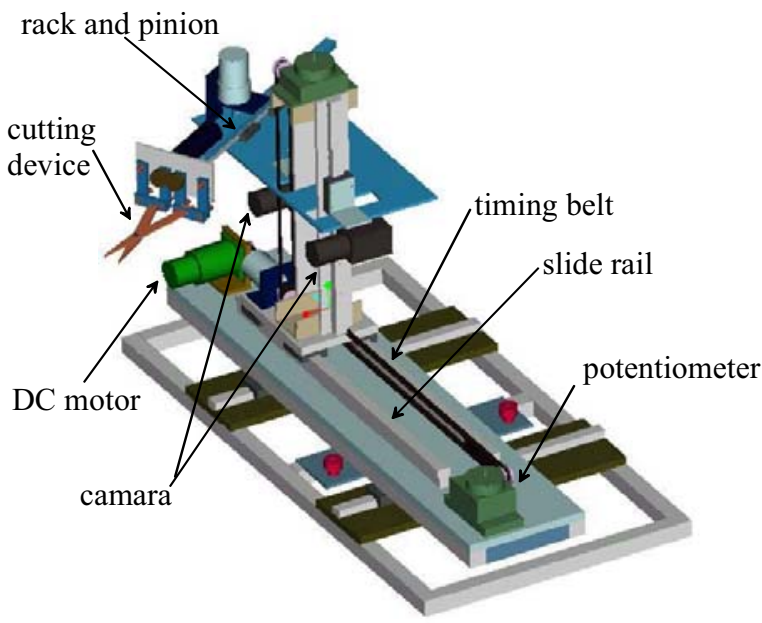

Fig.5 illustration of picking robot

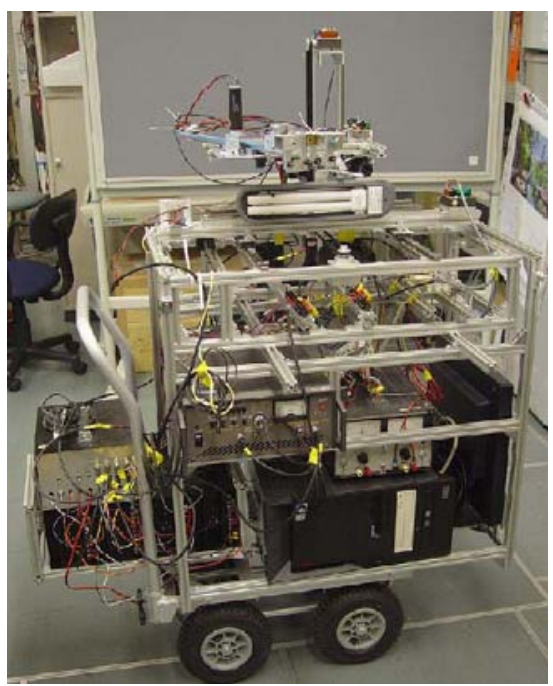

Fig.6 Prototype picking robot 
PC

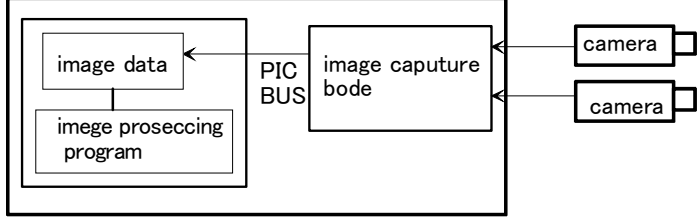

Fig. 7 image processing system

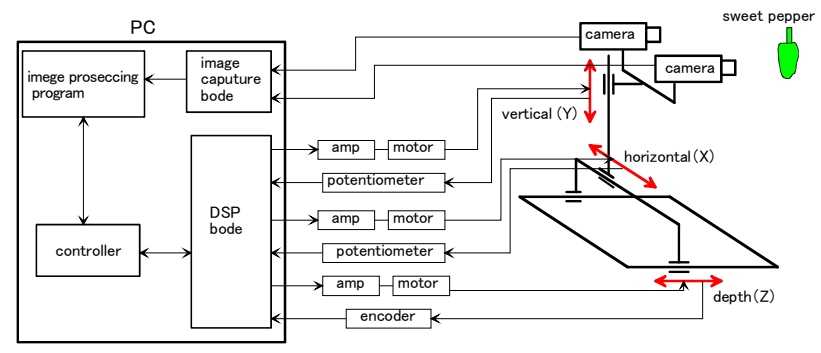

Fig.8 camera positioning system

\section{Recognition}

1) Image processing algorism: In the image-processing algorithm, the fruit of the sweet pepper is recognized by binarization of HSI color specification. HSI color specification system is one of color image expressions, it consist of three images that are hue, saturation, and intensity. The flow chart of recognition algorism is shown in Fig. 10 and Fig.11. However, this algorism cannot recognize fruits in the image with leaves as shown in Fig.12(c), because the color of fruits is almost same color of leaves. For identifying a fruit from leaves, this system need lighting for peppers. When sweet peppers are lighted, the grey values of fruit are different from it of leaves in the saturation of HIS as shown in Fig.12 (d). Only fruit part can be recognized by this algorism and lighting in the image. Serial of the processing images of the proposed method are shown as Fig.13.

2) Stereovision: Stereovision system of two cameras that measure the distance between the camera and the sweet pepper is illustrated as shown in Fig.9. The coordinates of a sweet pepper in the left and the right captured images are different. The distance $d$ is calculated from the substraction $(x l-x r)$ as

$$
d=b f /(x \ell-x r)
$$

where $f$ is focal length, and $d$ is distance of two cameras [4]. The horizontal and vertical position of the fruit of the sweet pepper can be measured from the image directly. Consequently, the 3D information of the fruit could be obtained

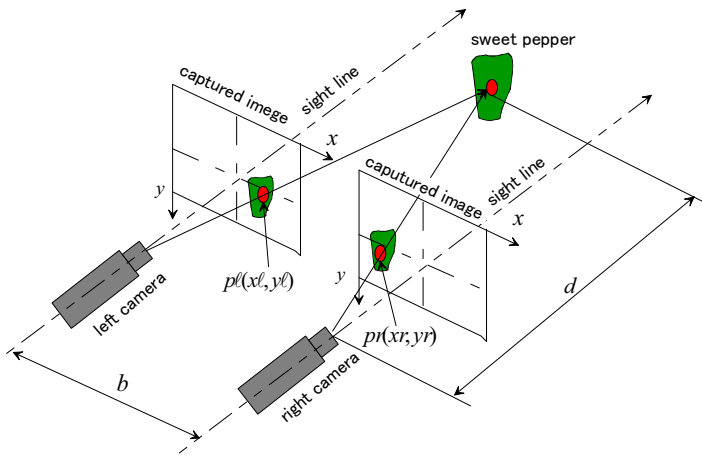

Fig.9 Parallel stereovision

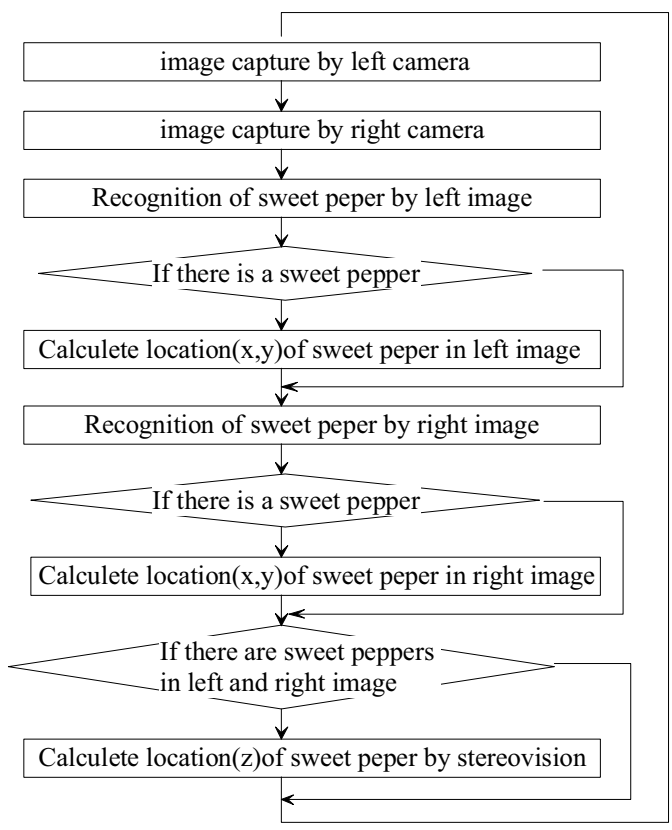

Fig.10 flow chart of image possessing

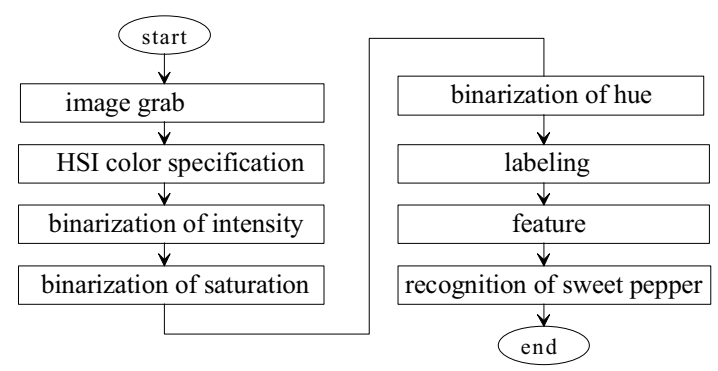

Fig.11 image processing algorism for recognition of sweet pepper 


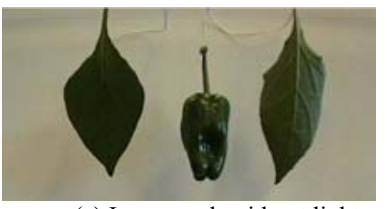

(a) Image grab without light

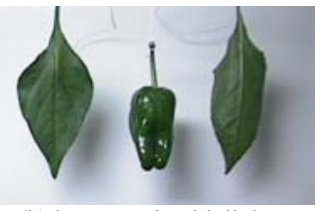

(b) image grab with light

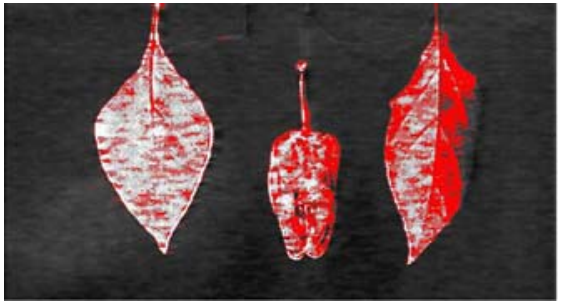

(c) Grey value without light

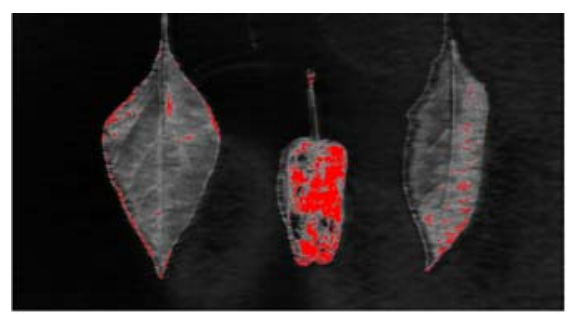

(d) Grey value with light

Fig.12 Change of grey value by light

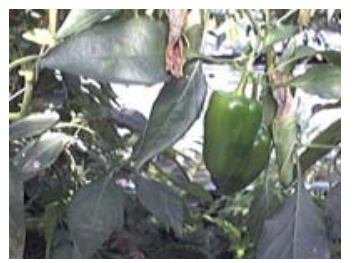

(a) Image grabs

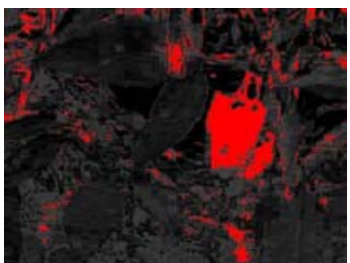

(c) Binarization (saturation)

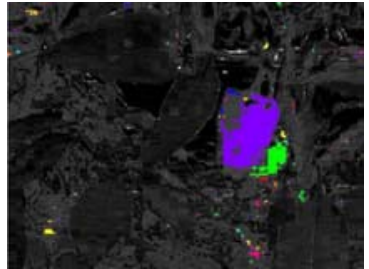

(c) Labelling

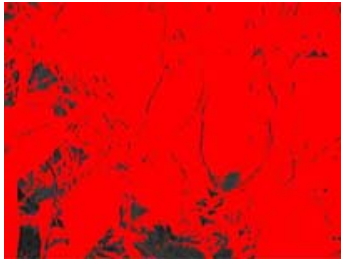

(b) Binarization (intensity)

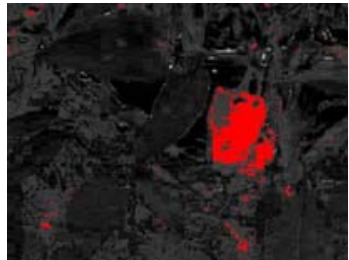

(b) Binarization (Hue)

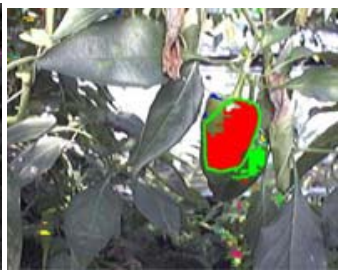

(d) Feature quantity
Fig.13 Example of image processing

\section{Camera positioning system}

The positioning system of cameras controls three actuators for the frame which has a cutting device and cameras shown in Fig. 5 and uses visual feedback control. The block diagram of visual feedback control is shown in Fig.14. The system is operated as follows. First, two CCD cameras move around with capturing the color images as a fruit of a sweet pepper is recognized on both images. After the fruit has been caught, the center of the fruit is made to align to the reference center as shown in Fig.15. Last, the depth position is calculated from the center positions of the both recognized fruit images, and actuates the frame in the depth direction until reference depth position.

\section{E. Cutting device}

Photos of the cutting device are shown in Fig.16. We use parallel linkage mechanism as shown in fig. 17 and a pruner. This pruner can be opened and closed by the DC motor control. The pruner is a usual one, and is easily and attached removed by the parallel linkage. Consequently, the pruner is easily changeable, as it has an ability to keep cutting performance sharply by grinding.

The cutting device is set on a translational link, which is driven by rack and pinion as shown in Fig5. After the camera has positioned for the recognized pepper, the link extends and the cutting device is approached for the pepper and cut it.

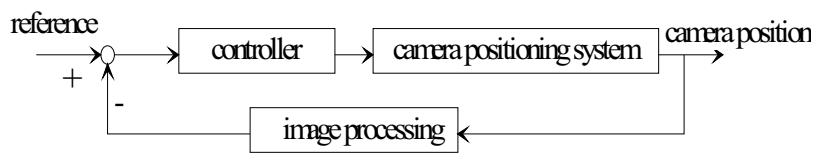

Fig.14 Visual feedback control

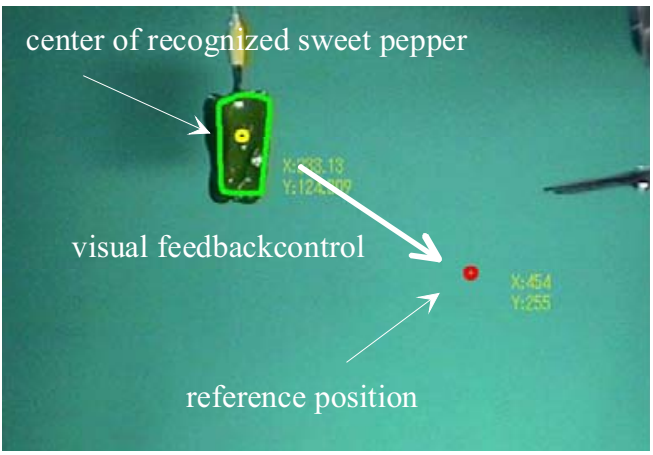

Fig.15 Visual feedback control in image 


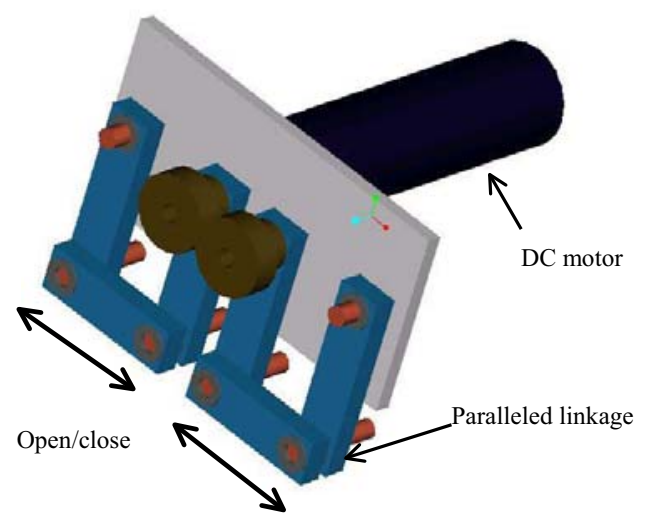

Fig.16 paralleled linkage mechanism

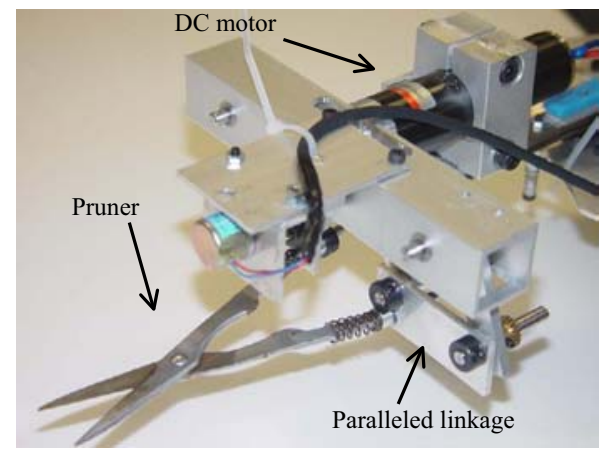

(a) Open the pruner

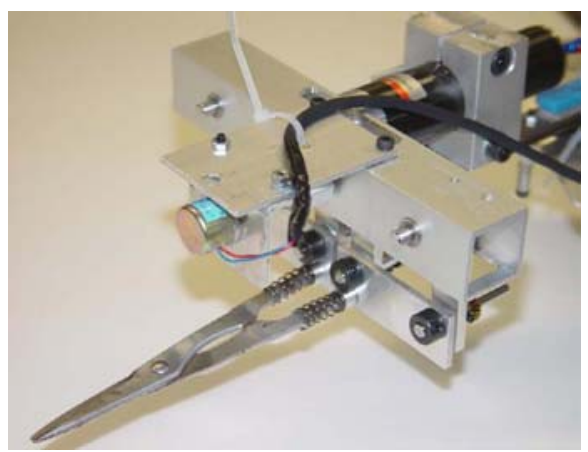

(b) Close the pruner

Fig.17 Picking device

\section{EXPERIMENT AND RESULT}

Experiments were carried out in two cases. One is without leaves as shown in Fig.19 and Fig.21, anther is with leaves as shown in Fig.20. The flow chart of the picking operation is shown in Fig. 18.
In the experiment without leaves, the sweet pepper was set in front of the picking robot as shown in Fig.19. First, two cameras moved in the horizontal and vertical, to look for the sweet pepper by the image processing (Fig.21 (a)). When the left camera recognized the sweet pepper, the left camera was controlled to positioning to the recognized sweet pepper using the visual feedback as come to the center position of the image. After the positioning in the two directions converged, the camera positioned to the sweet pepper in the depth direction with the stereovision method. When the positioning in the depth direction finished, the link is slid and the cutting device is approaching to the stem, and cut it as shown in Fig.21(c) and Fig.21 (d). After the cutting of stem, this robot returns to the initial position. In this case, the recognition of the fruit and the cutting of the stem went well. Another sweet peppers operation also went well.

In experiment with leaves, all of sweet peppers shown in Fig.20 could be were recognized, and the fruits of the left side and center could be cut. But the fruit of the right side was failed in cutting, because leaves covered the stem where the pruner would cut.

\section{CONCLUSION}

A picking robot for sweet peppers in greenhouse horticulture has been developed. In this paper, the developed prototype robot has been explained about functions and each part, and the results of the experiments have been shown. The functions roughly divided into the recognize system for the fruit of sweet peppers and cutting system. The robot has recognition system and cutting system. In the recognition system, we used image processing with lighting and stereovision. In the cutting system, we developed the camera positioning system with visual feedback control and cutting device. In addition, we carried out experiments in two simple situations in the laboratory. First experiment was carried out without leaves, and recognition of the fruit and cutting the stem was succeeded. Second experiment was carried out with leaves. In the case, recognition was succeeded, but success rate of cutting the stem became low because leave covered the stem. The improvement of ability to recognize the fruit rate and the cutting system will be studied in the future research.

\section{REFERENCES}

[1] S.Arima, S.Yuki, J.Yamashita, T.Katou, K.Marumi, "Studies on Harvesting Robot for Strawberry Grown on Annual Hill Top," ROBMEC'03, 1P1-2F-A5, 2003.

[2] M.Morita, K.Namba, T.Nishi, "Teleperrating Robotics in Agriculture," ROBMEC'03, 1P1-2F-A1, 2003.

[3] K.Oka, S.Kitamura, "Picking Robot in Green House -A Study of Image Recognition for Sweet pepper-," FAN Symposium'04, pp.317-318, 2004.

[4] N.Suganura, N.Fujiwara, K.Senda, "Lane Marker Extraction on the Highway Using Stereovision System and Active Contour Model," ROBMEC'03, 2A1-1F-C3, 2003. 


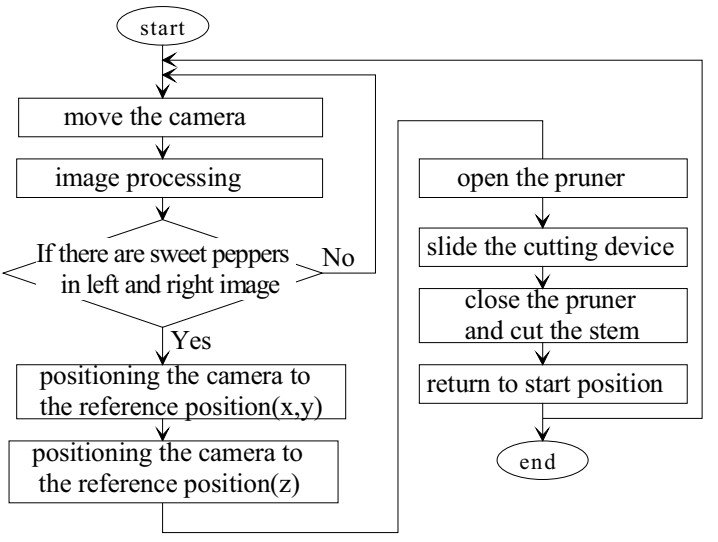

Fig.18 Flow chart of picking system

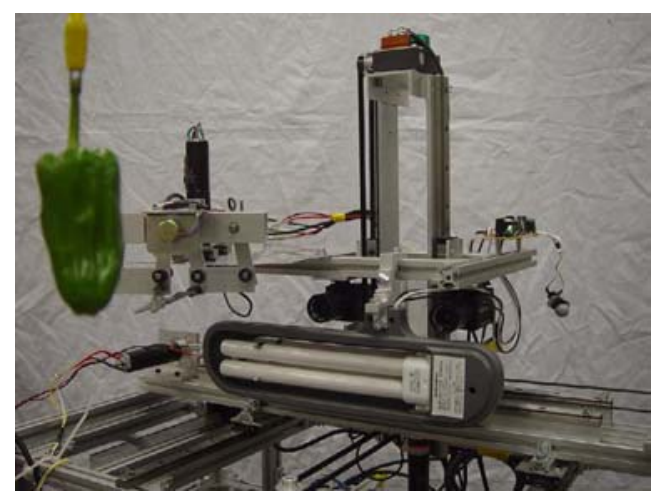

Fig.19 Experiment of picking robot without leaves

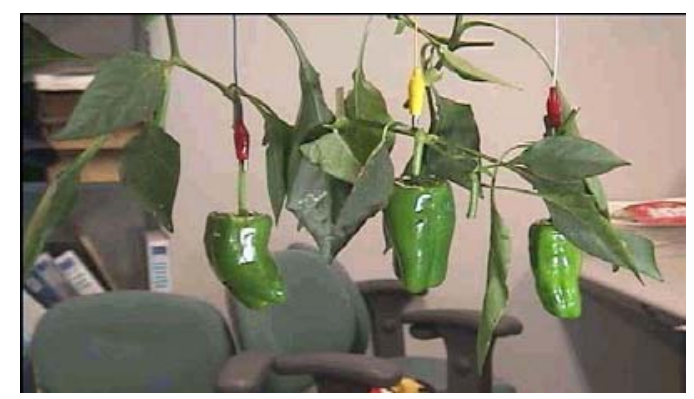

Fig.20 Experiment of picking robot with leaves

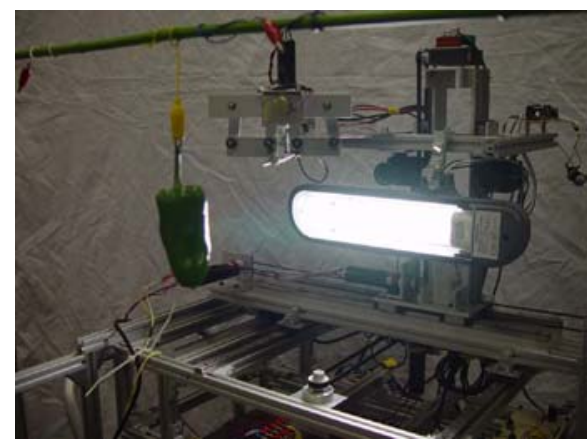

(a)

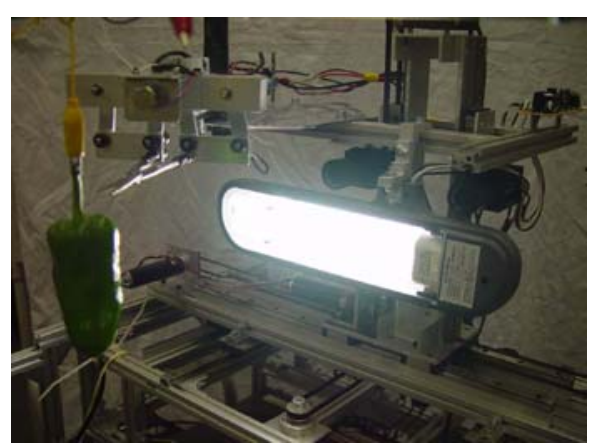

(b)

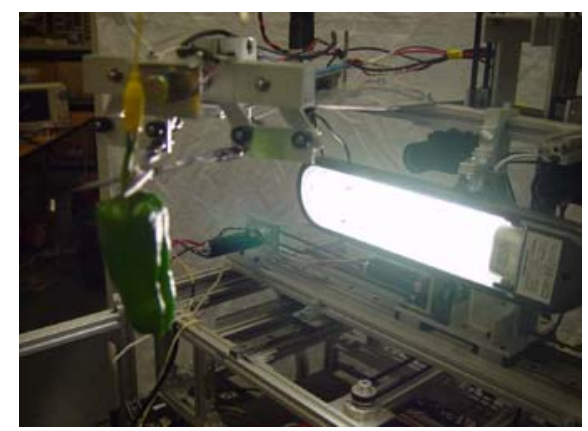

(c)

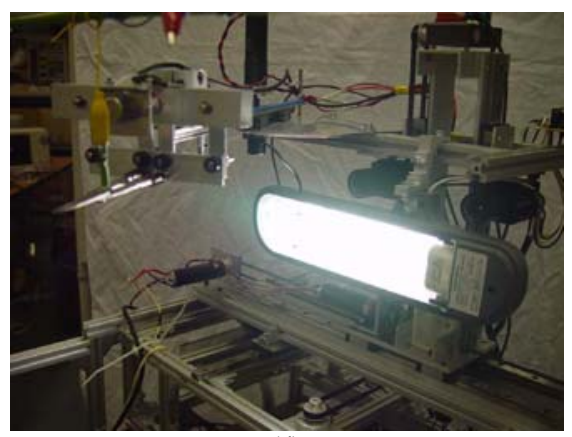

(d)

Fig.21 Result of experiment without leaves 